\title{
Models of the Cartwheel Galaxy
}

\author{
A. Bosma \\ Observatoire de Marseille, 2 Place Le Verrier, 13248 Marseille Cedex 4, \\ FRANCE
}

\begin{abstract}
I will briefly describe ongoing work on numerical simulations intended to reproduce the Cartwheel galaxy, a prototype ring galaxy located in a compact group of galaxies.
\end{abstract}

\section{Introduction}

The Cartwheel Galaxy is one of the best known prototypes of a small class of galaxies called ring galaxies. Such galaxies are thought to have been produced as a result of a well targeted collision during which a compact intruder galaxy crashes through the central parts of a disk galaxy (Theys \& Spiegel 1976, 1977; Lynds \& Toomre 1976). The ring itself is seen as the crest of a high amplitude density wave, which sweeps up the gas in the target disk, causing massive star formation on an organized scale. For a extensive review of these galaxies, see Appleton \& Struck-Marcell (1996).

A picture of the group of galaxies containing the Cartwheel is shown in Figure 1, where we indicate the Cartwheel itself and its companions labelled G1, G2 and G3. The group is in fact a compact group, SGG 0035-3357 (Iovino et al., in prep.). Early work by Fosbury \& Hawarden (1977) indicates that the outer ring of the Cartwheel is expanding, and that it shows the spectroscopic signatures of a burst of star formation in a low metallicity environment. Davies \& Morton (1982) measured the stellar velocity dispersion of the companion galaxy $\mathrm{G} 2$, and suggest that its relatively low mass might not have been sufficient to have done the damage to the Cartwheel.

Recently, a wealth of new data have been obtained for the Cartwheel and its companions. Marcum et al. (1992) have measured K-band magnitudes of the 4 galaxies in the group. Higdon $(1995,1996)$ presents an extensive study of the radio-continuum and detailed $21-\mathrm{cm} \mathrm{H}$ I line data of the galaxies in the group. He finds that the outer ring of the Cartwheel contains most of the $\mathrm{H}$ I gas in that galaxy. The magellanic irregular G1 seems to be relatively undisturbed, and weak $\mathrm{H}$ I emission is detected in the companion G3. A faint "bridge" seems to link the Cartwheel with G3, thus inciting Higdon (1996) to suggest that G3 was the intruder. $\mathrm{H} \alpha$-kinematics of the Cartwheel and of G1 have been presented by Amram et al. (1997) using a Fabry-Pérot scanner. They find only modest agreement with the data by Higdon (1996), suggesting that the $\mathrm{H} \alpha$ - and H I-kinematics may be different.

Complementary to these kinematical data, Struck et al. (1996) obtained a spectacular image of the Cartwheel and its close companions with the Hubble 
Space Telescope. In it, the central parts including the inner ring appear to have older populations, while the regions of the spokes and the outer ring appear to be much bluer. Nevertheless, the spokes contain little $\mathrm{H} \alpha$ emission (cf. Amram et al. 1997). Charmandaris et al. (1998) present ISOCAM images at 7 and $15 \mu \mathrm{m}$, and show that there is a hot spot in the outer ring in the Cartwheel, where star formation seems to be particularly prominent. This same region is also the site of X-ray emission, as reported by Wolter et al. (1999). Horellou et al. (1998) detect CO in the central regions of the Cartwheel. Although the signal is weak, they derive a systemic velocity of the Cartwheel of $9130 \pm 17$ $\mathrm{km} \mathrm{s}^{-1}$, to be compared with the H I determination by Higdon (1996) of 9089 $\pm 5 \mathrm{~km} \mathrm{~s}^{-1}$, and the $\mathrm{H} \alpha$ determination by Amram et al. (1997) of $9050 \pm 3$ $\mathrm{km} \mathrm{s}^{-1}$. Clearly these discrepancies make the modelling more difficult, since the projected velocity difference between the intruder and the target might help constrain the possible models.

\section{Numerical simulations}

Why model the Cartwheel in detail, since the principal physics of the encounter seems to be well established? One reason is that it is perhaps possible to date the starburst in the outer ring once the correct orbit of the intruder is established. Another is to do "accident reconstruction", i.e. to try to find out the most likely parameters of collision, such as the mass ratios and the likely orbit of the companion. A third is related to the mystery of the spokes. Normally one would expect them to be able to retain a lot of gas, and indeed one even expects them to be gas rich, yet they show no appreciable $\mathrm{H} \alpha$ emission. Thus somehow star formation does not take place at a prodigious rate in the spokes, contrary to the situation in the outer ring. Something in the problem still seems to be entirely unclear.

The new observations also pose the question which of the companions was the intruder. Since G1 is a relatively undisturbed magellanic irregular, it seems an unlikely candidate. Such loosely bound galaxies may not survive the violent impact. A better candidate seems G2, which was thought to be an elliptical, but on the HST image it looks like an S0 galaxy with a stellar 2-armed spiral structure. A faint extension, however, betrays a sign of interaction. Finally, since the work of Higdon (1996), G3 seems a candidate as well. As for the mass ratios between the intruder and the target galaxy, the best bet seems to be to rely on the K-band data. As shown in Table 1, the ratio of K-band magnitudes is such that the mass ratio of the Cartwheel disk is about the same as the total visible mass of either G2 or G3.

We are currently modelling the interaction of the Cartwheel group using $\mathrm{N}$-body simulations on dedicated GRAPE hardware. A satellite modelled by a Plummer sphere is thrown at a target disk galaxy modelled by a Plummer sphere bulge, a Kuzmin-Toomre disk and a Plummer sphere halo. Typically the target galaxy consists of 200000 particles: 10000 for the bulge, 40000 for the disc, and 150000 for the halo, while the satellite is taken to have 35 000 particles. The mass ratios for the "visible" parts - disc + bulge of the target, and (part of) the satellite - are thus in rough agreement with those inferred from available infrared data. The models (precursors are published in 


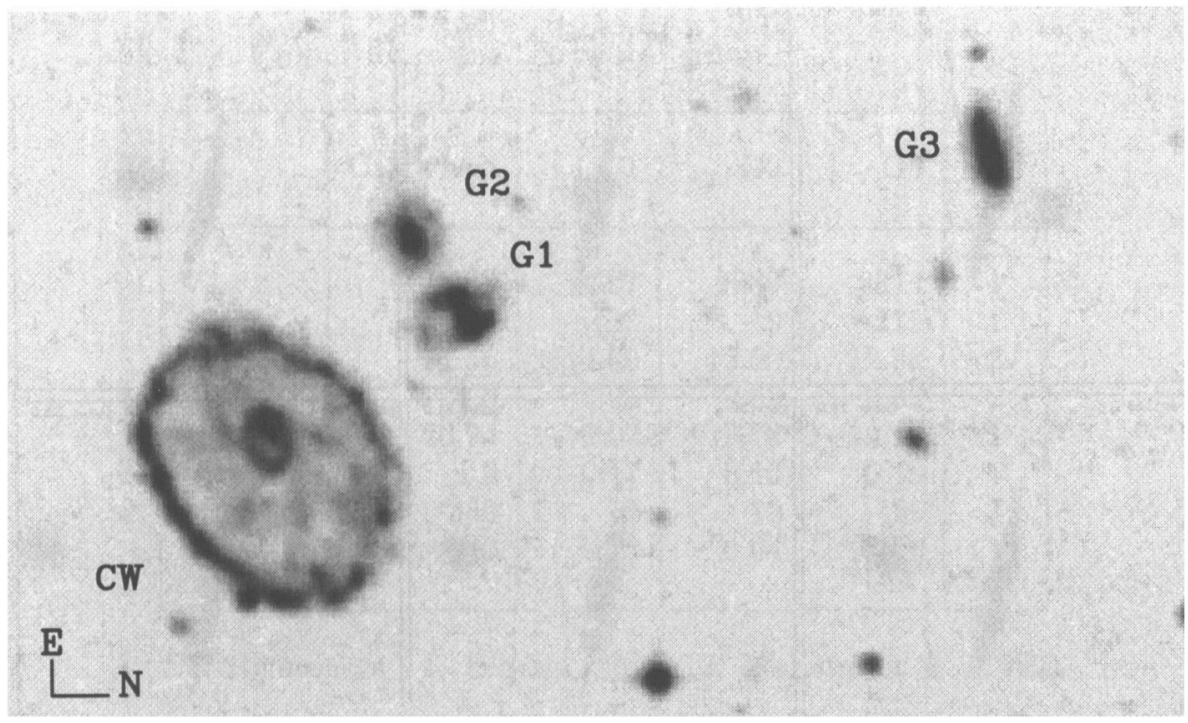

Figure 1. The group of galaxies SGG 0035-3357, comprising the Cartwheel and its companions G1, G2 and G3 (image from the DSS).

Athanassoula et al. 1997) reproduce several features of the collision, including the formation of the rings and the spokes, but a better match to all of the observations should still be possible.

\section{A "typical simulation"}

We have concentrated on matching the observations, assuming G2 is the intruder. The reason for this is simplicity : only if we cannot match the data completely with G2 as the intruder, then we will concentrate on G3. Fully centered collisions will not create enough asymmetry : the outer ring of the Cartwheel is well fitted by a circle seen under the inclination of $42^{\circ}$, but the center of this circle does not coincide with the center of the bulge. To create asymmetry, we will thus offcenter the collision slightly.

As for the spokes, their number comes out to be 7, as observed. Since we consider the spokes as transient wakes, we have not tried to create initial conditions which generate a different number of spokes.

An edge-on view of a sequence of snapshots is shown in Figure 2. This sequence is close to the best match we have so far with G2. It can be seen that the expanding ring is not entirely in the same plane as the initial disc. This might explain the imperfect agreement between the $\mathrm{H} \alpha$ and $\mathrm{H}$ I velocity field.

The "current" snapshot, i.e. the one corresponding to the observed situation, occurs at $t=29$, and fits reasonably well, as is shown in Figure 3 . As for the internal velocities, we can scale our simulations, and find a behaviour for 


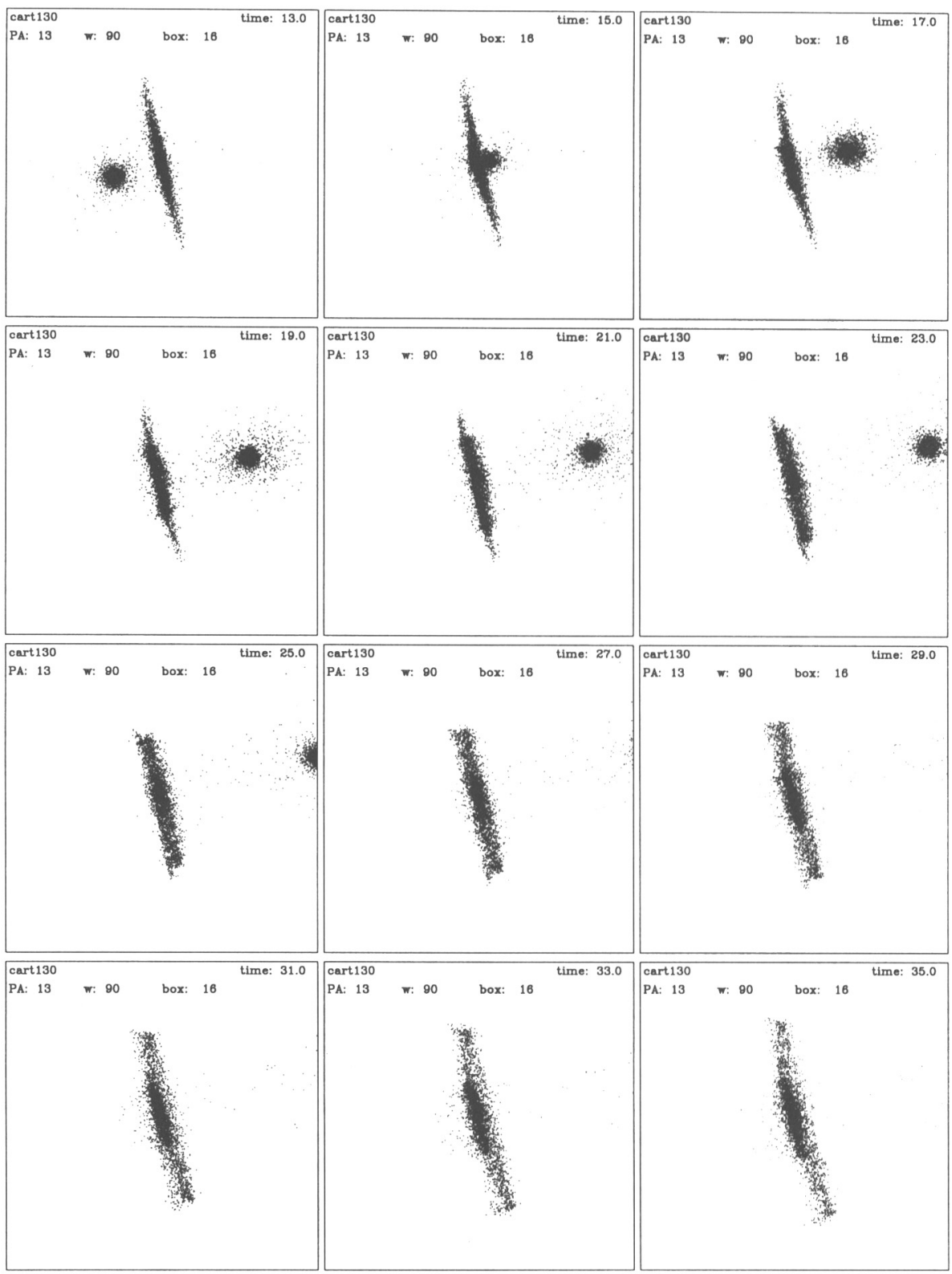

Figure 2. Edge-on view of the collision, illustrating the thickening of the target galaxy with time. 
Table 1. Properties of the Cartwheel group

\begin{tabular}{|l|r|r|r|r|l|}
\hline & Cartwheel & $\mathrm{G} 1$ & $\mathrm{G} 2$ & $\mathrm{G} 3$ & ref. $^{a}$ \\
\hline $\mathrm{V}_{\text {sys }}$ & 9089 & 8667 & 9080 & 8874 & 1 \\
$\Delta \mathrm{V}$ & 0 & -422 & -9 & -215 & 1 \\
$\mathrm{~K}$ magn. & 11.55 & 14.80 & 12.50 & 12.80 & 2 \\
$\mathrm{~K}$ ratio & 1.0 & 0.05 & 0.42 & 0.32 & 2 \\
$\mathrm{M}_{B}$ & -20.94 & -19.02 & -18.72 & -19.16 & 1 \\
$\mathrm{~B}$ ratio & 1.0 & 0.17 & 0.13 & 0.19 & 1 \\
$\mathrm{M}_{\text {ind }}$ & 340 & 11 & 12 & 26 & 1 \\
ratio & 1.0 & 0.03 & 0.04 & 0.08 & 1 \\
\hline
\end{tabular}

${ }^{a}$ References : 1. Higdon (1996), 2. Marcum et al. (1992)

the "rotation curve" and the expansion velocities as function of radius which is quite similar to the observations described in Fig. 11 of Higdon (1996).

\section{Discussion}

Several basic parameters of the satellites are still rather uncertain, and even the data for the Cartwheel itself are not as good as desired. The models show that the disk of the Cartwheel thickens after the passage, and that the companion looses part of its mass. It is thus necessary to a) study in detail the dynamical state of the 2 candidate intruders, for which hardly any information is available, and b) study the dynamical state of the stellar part of the Cartwheel, since the current data pertain mostly to the gaseous tracers, $\mathrm{HI}$ and $\mathrm{H} \alpha$.

Currently, for these companions, only a global estimate of their mass is available : for G2, the compact red companion close to the Cartwheel, only a central velocity dispersion is available from Davies and Morton (1982), while for the more distant G3 companion, some information is available from $21-\mathrm{cm}$ line data (Higdon 1996). New data on the stellar and gas kinematics of the Cartwheel itself should help us in several ways : it would give us another estimate of the redshift - there is a discrepancy of about $40 \mathrm{~km} \mathrm{~s}^{-1}$ between the $\mathrm{H} \alpha$ and the $\mathrm{H} \mathrm{I}$ systemic velocity, which in turn gives a big uncertainty in the relative velocities between the Cartwheel and its intruder - as well as a good estimate on the mass profile in the central parts.

Acknowledgments. Thanks are due to Lia Athanassoula and Ivanio Puerari for stimulating discussions on this work, and for permission to show results in advañce of publication. 


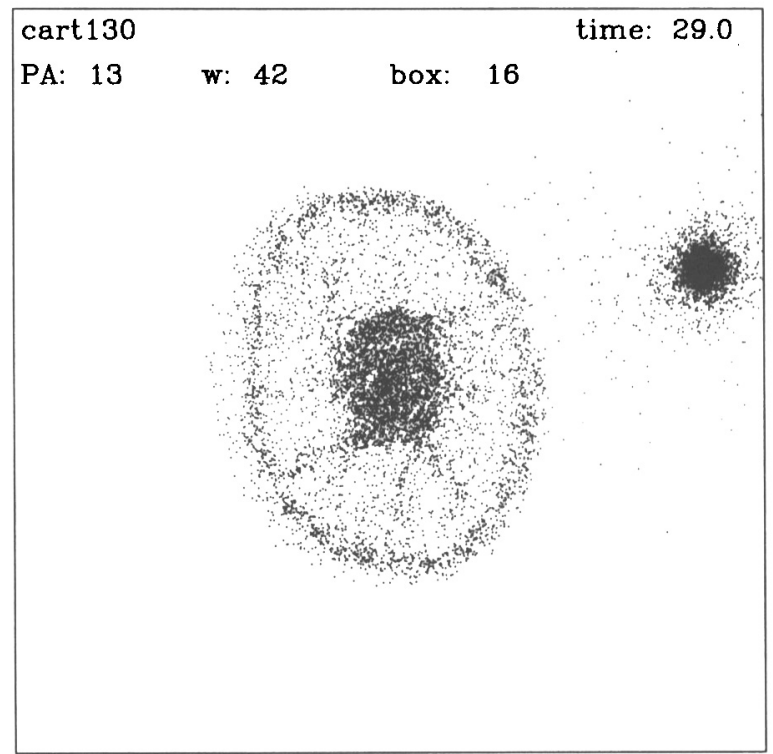

Figure 3. Snapshot of the collision at the moment of closest resemblance with the Cartwheel. Note that the fit is not yet perfect.

\section{References}

Amram, P., Mendes de Oliviera, C., Boulesteix, J., Balkowski, C. 1998, A\&A, 330,881

Appleton, P. N., Struck-Marcell, C. 1996, Fund. Cosm. Phys., 16, 111

Athanassoula, E., Puerari, I., Bosma, A. 1997, MNRAS, 286, 284

Charmandaris, V., Laurent, O., Mirabel, I. F., Gallais, P., Sauvage, M., Vigroux, L., Cesarsky, C., Appleton, P. N. 1999, A\&A, 341, 69

Davies, R. L., Morton, D. C. 1982, MNRAS, 201, 69P

Fosbury, R. A. E., Hawarden, T. G. 1977, MNRAS, 178, 473

Higdon, J. L. 1995, ApJ, 455, 524

Higdon, J. L. 1996, ApJ, 467, 241

Horellou, C., Charmandaris, V., Combes, F., Appleton, P. N., Casoli, F., Mirabel, I. F. 1998, A\&A, 340, L51

Lynds, R., Toomre, A., 1976, ApJ, 209, 382

Marcum, P. M., Appleton, P. N., Higdon, J. L. 1992, ApJ, 399, 57

Struck, C., Appleton, P. N., Borne, K. D., Lucas, R. A. 1996, AJ, 112, 1868

Theys, J., Spiegel, E. A., 1976, ApJ, 208, 650

Theys, J., Spiegel, E. A., 1977, ApJ, 212, 616

Wolter, A., Trinchieri, G., Iovino, A. 1999, A\&A, 342, 41 